\title{
Exact parametric solutions of the two members Emden-Fowler type nonlinear ODE $y_{x x}^{\prime \prime}=A x^{n_{1}} y^{m_{1}}+B x^{n_{2}} y^{m_{2}}$
}

\author{
D.E. Panayotounakos, Th. Zarmpoutis* and A. Kostogiannis \\ Division of Mechanics, Department of Applied Mathematical and Physical Science \\ National Technical University of Athens, Athens, Greece \\ *Corresponding author E-mail: zarmpoutis@tee.gr
}

\begin{abstract}
A mathematical solution methodology in combination with the mathematical technique concerning the construction of exact parametric solutions of the two member Emden-Fowler nonlinear ordinary differential equations (ODE) $y_{n}^{\prime \prime}=A x^{\prime \prime \prime} y^{\prime \prime}+B x^{n} y^{\prime \prime \prime} ; \quad A \neq B \neq 0$, are developed . Our main mathematical tools are the introduction of successful functional transformations and substitutions with convenient ad hoc assumptions in accordance with the problem being examined.
\end{abstract}

Keywords: Two members Emden-Fowler nonlinear ODEs; exact parametric solutions; convenient ad hoc assumptions.

\section{Introduction}

In the present work we provide and develop a new mathematical methodology together with the corresponding solution technique concerning a two member Emden-Fowler nonlinear ODE $y_{x x}^{\prime \prime}=A x^{n_{1}} y^{m_{1}}+B x^{n_{2}} y^{m_{2}}$, where $A \neq B \neq 0$, and $n_{1}, m_{1}, n_{2}, m_{2}$ are given exponents. In [7] the number of all solvable types of equations of the above form are presented and tabulated. The two parameter families (in the space of parameters and $n_{2}, m_{2}$ ), the one parameter families and isolated points are represented in a consecutive form. The equations are arranged in accordance with the growth of $m_{1}$, the growth of $m_{2}$ (for indentidal $m_{1}$ ), the growth of $n_{1}$ ( for indentical $m_{1}$ and $m_{2}$ ) and the growth of $n_{2}$ ( for indentical $m_{1}, m_{2}$ and $n_{1}$ ). On the other hand in [4,6,9] some classes of nonlinear Volterra and Emden-Fowler equations were solved by using the discrete group method. Also the mathrmatical methodology developed in [1] provides a very fruitful means in solving such kinds of ODEs. Moreover, Bäcklund transformations, having their origins in differential geometry (the last nontrivial example is the transformation of pseudospherical surfaces introduced by L. Bianchi and A.V. Bäcklund in 1880) are a useful tool in soliton theory and integrable systems govering by such systems [8]. Use of Bäcklund transformations is presented in [7]. However, they were applied in concrete number of ODEs which are also tabulated.

In the present work through convenient admissible functional transformations and substitutions, the above mentioned nonlinear ODE $y_{x x}^{\prime \prime}=A x^{n_{1}} y^{m_{1}}+B x^{n_{2}} y^{m_{2}},\left(A, B \neq 0 ; n_{1}, m_{1}, n_{2}, m_{2}=\right.$ arbitrary $)$ can be reduced to an Abel nonlinear ODE of the second kind of variable coefficients (smooth functions of a parameter $u$ ), that must be defined. Relative transformations are included in [3,5;p. 27 (b),7]. Using some results developed in [5; 5;p. 27 (b),7], we introduce new convenient functional transformations and substitutions so that the beforementioned coefficients are defined so that finally to be possible the constructions of exact parametric solutions concerning the two member Emden-Fowler nonlinear ODE under consideration. The application being examined concerns the simple Emden-Fowler equation $y_{x x}^{\prime \prime}==A x^{n} y^{m} ; A \neq B \neq 0, n, m$ arbitrary components and the obtained exact parametric solutions cover at least the cases tabulated in [7].

The introduced mathematical methodology and the developed solution technique are general and can be applied to a wide number of classes of nonlinear ODEs appearing in mathematical physics and nonlinear mechanics. 


\section{Some basic results}

Before we address the issue of exact parametric solutions for the Emden-Fowler type nonlinear ODE we will remind a digression of known exact solutions of some classes of ODEs and we will provide admissible functional transformations and substitutions that reduce a class of ODEs to different class.

An Abel equation of the second kind is of the type

$$
\left[g_{1}(x) y+g_{0}(x)\right] y_{x}^{\prime}=f_{2}(x) y^{2}+f_{1}(x) y+f_{0}(x), g_{1}(x) \neq 0
$$

There are suitable admissible functional transformations $[3,7]$ that reduce equation $(2.1)$ to the canonical (normal form)

$y y_{x}^{\prime}-y=F(x), \quad F(x)=$ free smooth member.

We dispose the following $[5,7 ;$ p.27 (b)]

Theorem 2.1 If the smooth variable coefficients of the Able equation (2.1) satisfy the functional relation [5; p. 27 (b),7]

$g_{0}\left(2 f_{2}+g_{1_{x}}^{\prime}\right)=g_{1}\left(f_{1}+g_{0_{x}}^{\prime}\right), \quad g_{1} \neq 0$,

then its general solution if given by the formula

$$
\frac{g_{1} y^{2}+2 g_{0} y}{g_{1} J}=2 \int \frac{f_{0}}{g_{1} J} d x+C,
$$

where the factor $J(x)$ is given by

$$
J(x)=\exp \int \frac{2 f_{2}}{g_{1}} d x, \quad g_{1}(x) \neq 0 .
$$

A generalized Emden-Fowler equation is of the type [7]

$$
y_{x x}^{\prime \prime}=A x^{n} y^{m}\left(y_{x}^{\prime}\right)^{1}
$$

The case $l=0$ corresponds to the classical Emden-Fowler equation. For several details for these equations. For the triad of exponents $\{n, m, l\} \neq\{0,0,0\}$ one reads the following

Theorem 2.2 The substitution

$$
z=\frac{x}{y} y_{x}^{\prime}, U=A x^{n-l+2} y^{m+l-1} ; y \neq 0
$$

reduces the generalized Emden-Folwer equation (2.5) to the equation

$$
\left(z^{l} U-z^{2}+z\right) U_{z}^{\prime}=(m+l-2) z+[(n-l+2)] U,
$$

that further through the new substitution

$\xi=U-z^{2-l}+z^{1-l}$

guides to the Abel equation of the second kind

$$
\begin{gathered}
\xi \xi_{z}^{\prime}=[(m+2 l-3) z+(n-2 l+3)] z^{-1} \xi+ \\
+\left[(m+l-1) z^{2}(n-m-2 l+3) z-m+l-2\right] z^{1-2 l} .
\end{gathered}
$$


The fourth class of Emden-Fowler type of equations which will be examined in the following, is that of a two member Emden-Folwer equations of the form

$$
y_{x x}^{\prime \prime}=A x^{n_{1}} y^{m_{1}}+B x^{n_{2}} y^{m_{2}} \text {. }
$$

The two-parameter families (in the space of parameters $m_{1}, m_{2}, n_{1}$ and $n_{2}$ ), the one parameter families and isolated points are represented in a consecutive fashion in [7]. Equations are arranged in accordance with the growth of $m_{1}$, the growth of $m_{2}$ (for indentidal $m_{1}$ ), the growth of $n_{1}$ ( for indentical $m_{1}$ and $m_{2}$ ) and the growth of $n_{2}$ ( for indentical $m_{1}, m_{2}$ and $n_{1}$ ).

In the next section, we shall provide a new mathematical technique for solving parametrically such type of equations for arbitrary $m_{1}, m_{1}, n_{1}$ and $n_{2}$.

\section{New results}

We state the following

Proposition 3.1 For a given generalized Emden-Fowler type of nonlinear ODE

$y_{x x}^{\prime \prime}=A x^{n_{1}} y^{m_{1}}+B x^{n_{2}} y^{m_{2}}$;

$n_{1}, m_{1}, n_{2} m_{2}=$ arbitrary; $A \neq B \neq 0$,

there exist admissible co-ordinate transformations that can reduce it to an Abel nonlinear ODE of the second kind of variable coefficients.

Proof: We set

$U=A x^{n_{1}+2} y^{m_{1}-1}, z=\frac{x}{y} y_{x}^{\prime}$.

and we compute

$d U=\left[A\left(n_{1}+n_{2}\right) x^{n_{1}+1} y^{n_{1}-1}+A\left(m_{1}-1\right) x^{n_{1}+2} y^{n_{1}+2} y_{x}^{\prime}\right] d x$,

while using $(3.2 \mathrm{a}, \mathrm{b})$ we lead to the equation

$d U=\left[A\left(n_{1}+2\right) x^{n_{1}=1} y^{m_{1}-2}+A\left(m_{1}-1\right) x^{n_{1}+1} y^{m_{1}-2} \frac{z^{-1}}{y}\right] d y$,

or equivalently

$d u=A\left[\left(n_{1}+2\right)+\left(m_{1}-1\right) z\right] x^{n_{1}+1} y^{m_{1}-1} d x$

Also, by means of (3.2b), we estimate

$d z=\left[\frac{x}{y} y_{x x}^{\prime \prime}+\frac{y-x y_{x}^{\prime}}{y^{2}} y_{x}^{\prime}\right] d z=\left[\frac{x}{y}\left(A x^{n_{1}} y^{m_{1}}+B x^{n_{2}} B y^{m_{2}}\right)+\frac{y_{x}^{\prime}}{y} x\left(\frac{y_{x}^{\prime}}{y}\right)^{2}\right] d x=\frac{A x^{n_{1}+2} y^{m_{1}-1}+B x^{n_{2}+1} y^{m_{2}-1}+z(1-x z)}{x} d x$.

Here, we must notice that the system (3.2 a,b) is equivalent to the initial equation (3.1). Dividing by parts (3.3) and (3.4) one derives

$$
U_{z}^{\prime}=\frac{d U}{d z}=\frac{A\left[\left(n_{1}+2\right)+\left(m_{1}-1\right) z\right] x^{n_{1}+2} y^{m_{1}-1}}{A x^{n_{1}+2} y^{m_{1}-1}+B x^{n_{2}+1} y^{m_{2}-1}+z(1-x z)}=\frac{A\left[\left(n_{1}+2\right)+\left(m_{1}-1\right) z\right] U}{U+B x^{n_{2}+2} y^{m_{2}}+z(1-x z)} .
$$


In other words we perform an Abel type equation for $u$

$$
\left[U+B x^{n_{2}+2} y^{m_{2}}+z(1-x z)\right] U_{z}^{\prime}=A\left[\left(n_{1}+2\right)+\left(m_{1}-1\right) z\right] U
$$

By now making use of the ad hoc assumptions

$$
x=f(z), \quad y=g(z),
$$

where $f$ and $g$ are smooth functions to be determined, equations (3.6) becomes to the Abel equation of the second kind of variable coefficients

$$
\left[U+B f^{n_{2}+2} g^{m_{2}}+z(1-f z)\right] U_{z}^{\prime}=A\left[\left(n_{1}+2\right)+\left(m_{1}-1\right) z\right] U
$$

We continue by formulating the following

Corollary 3.2 For the Abel equation of the second kind

$\left[U+B f^{n_{2}+2} g^{m_{2}}+z(1-f z)\right] U_{z}^{\prime}=A\left[\left(n_{1}+2\right)+\left(m_{1}-1\right) z\right] U$

where $f(z)$ and $g(z)$ are smooth functions to be determined and $n_{1}, m_{1}, n_{2}, m_{2}$ are given factors, it is always possible to define $f(z)$ and $g(z)$ such that the considered equation admits an exact parametric solution.

Proof: Making use of the functional relation (2.3) given in Theorem 2.1 for the present case hold true

$$
\begin{aligned}
& (1-z) f(z)+\left(n_{1}+1\right) z+\left(n_{1}+z\right)=-\Omega(z) \Leftrightarrow f(z)=x=\frac{\left(n_{1}+1+\Omega(z)\right)+\left(n_{2}+2\right) z}{1-z}, z \neq 1 \\
& \left(f^{n_{2}+2}\right)_{z}^{\prime} g^{m_{2}}+m_{2}\left(g^{m_{2}}\right)_{z}^{\prime} f^{n_{2}+2}=\Omega(z) ;
\end{aligned}
$$

where $\Omega(z)$ is a arbitrary separation smooth function.

On the other hand formula (2.4) given in Theorem 2.1 in combination with (3.2a) given in Theorem 3.1 , admits the following general solution $U(z)[5 ; \mathrm{p} .27]$

$$
U=A x^{n+1} y^{m_{1}-1}=\left[B f^{n_{2}+2} g^{m_{2}}+z(1-f z)\right]\left\{1 \pm \sqrt{1+\left[\frac{2}{f^{n_{2}+2} g^{m_{2}}+z(1-f z)}\right]^{2}} C,\right.
$$

where $C$ is a first integration constant.

Moreover integration of (3.10) results [2]

$$
g^{m_{2}}(z)=C^{*} f^{-\left(n_{2}+2\right)}(z)+\frac{f^{-\left(n_{2}+2\right)}(z)}{m_{2} B} \int f^{-\left(n_{2}+2\right)}(z) \Omega(z) d z
$$

where $C^{*}$ is a second constant of integration. Simultaneously based on (3.7), performs us to write the functional relation

$$
1+C\left(\frac{2}{B f^{n_{2}+2} g^{m_{2}}+z(1-f z)}\right)=\left[\frac{A x^{n_{1}+1} y^{n_{1}-1}}{B f^{n_{2}+2} g^{m_{2}}+z(1-f z)}+1\right]^{2}
$$

The prove of the proposed Corollary 3.2 is now completely finished since by equations (3.9) to (3.11), one computes $\Omega(z), f(z), g(z)$ that is we define the separated arbitrary function $\Omega(z)$ as well as $x$ and $y$ in terms of the parameter $z$. The solution contains two constants of integrations $C$ and $C^{*}$. 
Summarizing the obtained results we dispose the following

Theorem 3.3 For a given two members Emden-Fowler ODE

$y_{x x}^{\prime \prime}=A x^{n_{1}} y^{m_{1}}+B x^{n_{2}} y^{m_{2}} ;(A, B \neq 0)$,

there exists always an exact nontrivial parametric solution given by the following equations

$1+C\left(\frac{2}{B f^{n_{2}+2} g^{m_{2}}+z(1-f z)}\right)=\left[\frac{A x^{n_{1}+1} y^{n_{1}-1}}{B f^{n_{2}+2} g^{m_{2}}+z(1-f z)}\right]^{2}$,

$x=\frac{\left[n_{1}+1+\Omega(z)\right]+\left(n_{2}+2\right) z}{1-z}$,

$y^{m_{2}}(z)=C^{*} x^{-\left(n_{2}+2\right)}(z)+\frac{x^{-\left(n_{2}+2\right)}(z)}{m_{2} B} \int x^{-\left(n_{2}+2\right)}(z) \Omega(z) d z ;$

$\Omega(z)=$ subsidiary smooth separation function to determined by the elimination;

$C, C^{*}=$ integration constants;

$-\infty<z=$ main parameter $<+\infty$.

The proof of this Theorem becomes by the Theorem 3.1 in combination with the Corollary 3.2. From now on the solution procedure that must be followed demands:

a. The solution of (3.9) furnishes $x$ in terms of $z$ and of the separate function $\Omega(z)$ that is

$x=f(z)=\frac{\left(n_{1}+1\right)(1+z)+\Omega(z)}{1-z}, z \neq 1$

b. Equation (3.10) permits us to estimate function $y$ in terms of $z$ and of the separate function $\Omega(z)$ by the integration of the linear ODE

$\left(f^{n_{2}+2}\right)_{z}^{\prime} g^{m_{2}}+m_{2}\left(g^{m_{2}}\right)_{z}^{\prime} f^{n_{2}+2}=\frac{\Omega(z)}{B} ;$

namely the linear ODE

$y=g(z)=\left[\frac{C^{*}-m_{2} \Omega(z)}{(n+1)(1+z)+\Omega(z)}\right],(n+1),(1+z) \neq \Omega(z)$,

c. The function $\Omega(z)$ can be estimated by the equation (3.12) that is the equation

$A^{2}\left(x^{n_{1}+1} y^{m_{1}-1}\right)+2 A B x^{n+1} y^{n_{1}-1}\left[x^{n_{2}+2} y^{m_{2}}+z(1-x z)\right]-2 C\left[B x^{n_{2}+2} y^{m+2}+z(1-x z)\right]=0$.

Inserting (3.14) and (3.15) into (3.16), we define $\Omega(z)$ as function of $z, A, B, C$ and $C^{*}$ where $C$ and $C^{*}$ are two integration constants.

\section{References}

[1] G. W. Bluman, J.D. Cole, The general similarity solution of the heat equation, J. Math. Mech., 18, (1969), pp. 1025-1042

[2] I.S Grandsteyn, I.M. Ryzhik, Table of Integrals, Series and Products, Academic Press, New York, 1965.

[3] G. JULIA, Exercises d'Analyse, B.d 3, Paris, 1935.

[4] M. Hadizadey, A.R. Zokayi, P. Darenia, On the dispute group analysis for solving some classes of Emden-Fowler equations, App. Math. Research, 5, (2004), pp. 169-178.

[5] E. Kamke, Differentialgleichungen, Lösungsmethoden und Lösungen, Vol. I, B. G. Teubneur, Stuttgart, 1977.

[6] L.S. Ousiannikov, Group analysis of differential equations, Academic Press, New York, 1982.

[7] A.D. Polyanin, V.F. Zaitsev, Handbook of Exact Solutions for Ordinary Differential Equations, CRC Press, New York, Second edition 2003.

[8] J. Satsuka, D. Kaud, A Bückland transformation for a higher order Korte-weg-de Vries equation, J. Physics Sc. Japan, 43, (1988), pp. 43-406.

[9] V.F. Zaitsev, On dicrete-group analysis of ordinary differential equations, Soc. Math. Dokl., 37, (1908), pp. 403-406. 